\title{
Current controversies: thrombolysis for patients with acute ischaemic stroke aged over 80
}

\author{
${ }^{1}$ GA Ford, ${ }^{2} \mathrm{P}$ Sandercock \\ ${ }^{1}$ Jacobson Chair of Clinical Pharmacology, Institute for Ageing and Health, Newcastle University, and Director, NIHR Stroke Research \\ Network, Royal Victoria Infirmary, Newcastle upon Tyne; ${ }^{2}$ Professor of Medical Neurology, University of Edinburgh, Edinburgh, UK
}

Stroke is the major cause of disability in the community. Most strokes are due to blocked arteries in the brain. Evidence is accumulating that clot-busting drugs improve outcome after ischaemic stroke. Recombinant tissue plasminogen activator ( $r t-P A)$ is licensed for the treatment of selected patients within three hours of acute ischaemic stroke in many parts of the world, and stroke services are being developed so that eligible patients can receive this treatment as soon as possible after the onset of stroke symptoms. However, thrombolysis can cause bleeding into the brain, so the treatment should only be given when the benefits outweigh the risks. Controversy still exists about the risks and benefits in certain groups of patients, and there is variation in practice between stroke physicians, reflecting these uncertainties.

The JRCPE editorial team is delighted that two leading experts have agreed to debate the pros and cons of thrombolysis in people over the age of 80 years; one of the areas where there is still variation in practice. We hope that readers will find this debate enlightening, entertaining and informative.

\section{KEYWORDS Cerebral ischaemia, old age, stroke, thrombolysis}

DECLARATION OF INTERESTS Prof. Ford has received honoraria for educational activities related to stroke, consultancy activities related to thrombolysis with alteplase and a research grant for a cognitive study in stroke from Boehringer Ingelheim.

\author{
Correspondence to \\ GA Ford, \\ Level 6, Leazes Wing, Royal Victoria \\ Infirmary, Newcastle upon Tyne \\ NEI 4LP, UK \\ tel. $+44(0) 1912228090$ \\ e-mail g.a.ford@newcastle.ac.uk \\ $P$ Sandercock, \\ Department of Clinical \\ Neurosciences, Western General \\ Hospital, Edinburgh EH4 2XU, UK \\ tel. +44 (0) I 3 I 5372928 \\ e-mail peter.sandercock@ed.ac.uk
}

\section{Let's get the evidence from trials!}

P Sandercock

\section{Introduction}

Thrombolytic therapy is increasingly used as a treatment for acute ischaemic stroke. Its adoption into clinical practice in the UK has been limited: about $1-2 \%$ of all patients with acute ischaemic stroke presenting to hospital currently receive this treatment.' However, it is not universally available, and there is a 'postcode lottery' element to the chance of receiving treatment. ${ }^{2}$ Is there an ageist element, too? The answer is yes: for a start, thrombolytic therapy is not currently licensed for patients aged $>80$ years in the European Union. The debate therefore hangs on a series of questions:

a) Is the lack of approval for treatment of the over $80 \mathrm{~s}$ based on sound evidence?

b) Does age $>80$ years really affect the risks of thrombolysis (chiefly of death and of major intracranial haemorrhage)?

c) Does age $>80$ years affect the likelihood of benefit (e.g. reopening the blocked cerebral artery, partial or complete reversal of the neurological deficit or reducing the risk of major cerebral hemisphere swelling with increased intracranial pressure)?

d) Even if the evidence is not robust, are people over 80 being treated 'off-label' in the UK and elsewhere? e) Is there any role for using specialist brain imaging to select those older patients most likely to benefit from treatment?

f) In the light of all these questions, is there a case for further randomised controlled trials in patients aged $>80$ years?

\section{Over 80 s are the world's fastest growing age group}

All over the world, living to the age of 90,100 or even older is no longer exceptional, and the 'older old' (people aged 80 years and over) are the world's fastest growing age group. ${ }^{3}$ By 2050, the number of people aged over 80 will increase several fold (with the estimated relative increases being greatest in the developing world); to 6 million in the UK, I3 million in Brazil and 120 million in China. ${ }^{3}$ Given this large - and steadily increasing - number of people aged over 80 , the question of whether they should be considered for thrombolytic therapy if they have a stroke comes into very sharp focus. If the current EU approval is adhered to strictly, such people are excluded from treatment by virtue of their age alone. So what should happen in the UK to the 30,000 or so people aged over 80 each year who have an acute ischaemic stroke? The question almost has a Shakespearean ring: over 80 with a stroke - to treat or not to treat...? 
What is the evidence for treatment being restricted to the under 80 s?

The only agent approved for thrombolysis in stroke is recombinant tissue plasminogen activator ( $r t-P A)$. There have been II randomised controlled trials of rt-PA for acute ischaemic stroke, which have recruited just under

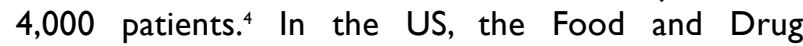
Administration approved rt-PA for acute stroke in 1995, but did not set an upper age limit for treatment (the US approval was based on a single trial of just 624 subjects). However, the European Medicines Agency (EMEA) was more cautious. The randomised trials that were completed at the time of the submission for approval for use in Europe included only 42 patients aged over 80 . In view of the lack of direct evidence on the effects in older people, the EMEA approved the treatment only for patients aged under 80 . Given the predicted shift to the population in the future, including many more older people, it is worrying that the evaluation of treatments for stroke in older people have been so underresearched. ${ }^{5}$ Thrombolysis for stroke is no exception!

\section{Does age affect the risk of death with treatment?}

The Cochrane systematic review of the randomised trials showed that, overall, rt-PA was associated with a non-significant $14 \%$ increase in the odds of death. ${ }^{4}$ A subgroup analysis of the effect of age on treatmentrelated death was not possible. ${ }^{4}$ The meta-analysis of individual patient data from all the available randomised trials could have examined whether the risk of death from treatment was modified by age, but, surprisingly, it did not. ${ }^{6}$ A systematic review of the non-randomised cohort studies comparing outcome after rt-PA treatment in patients aged under 80 , with patients aged over 80 , is of some interest. It included six cohort studies with 2,244 patients, of whom 477 were aged over 80 years. ${ }^{7}$ Since the studies were non-randomised, it is quite possible that the patients aged over 80 were systematically different to the patients aged under 80 . As one might expect, patients aged over 80 had a higher overall mortality, and seemed to have a higher chance of dying after treatment with rt-PA, although whether this was specifically an effect of the drug was unclear. ${ }^{7}$

\section{Does age affect the risk of intracranial haemorrhage} with treatment?

In the cohort studies, the risks of symptomatic intracranial haemorrhage (the most feared complication of thrombolytic therapy) were slightly, but not statistically significantly, higher in the older patients, although one could not exclude a substantially higher risk of bleeding in the older age group. ${ }^{7}$

\section{Does age affect the chance of benefit from rt-PA?}

The meta-analysis of individual patient data from all the available randomised trials could have examined whether the benefit from treatment was modified by age, but, again surprisingly, it did not. ${ }^{6}$ In the non-randomised cohort studies, the proportion who survived free of disability was smaller among the older patients compared with the patients under $80 .^{7}$ This is not to say that older people do not benefit at all from treatment, but perhaps that the scope for benefit is rather smaller than in younger people.

\section{Are people over 80 being treated with rt-PA?}

In the Hesse region of Germany, despite high treatment rates in younger patients, the percentage actually receiving treatment falls steeply with age. ${ }^{8}$ The same was true in a very large multicentre audit of hospitals in the US. ${ }^{9}$ For patients aged 80-89 years the odds of treatment with tissue plasminogen activator (tPA) were reduced by $60 \%$ and for patients aged over 90 years the odds of treatment were reduced by $80 \%{ }^{9}$ However, the rt-PA treatment included in the review by Engelter is clearly being used in the absence of EU approval in at least some patients over 80 , despite the lack of reliable evidence. ${ }^{7}$ Whatever else, if you are a patient with acute ischaemic stroke aged over 80 , your chance of receiving thrombolysis will depend very much on which country you are in and which hospital you are treated at. This is unacceptable.

\section{Is there a role for specialist brain imaging?}

The standard imaging modality for patients with acute stroke being considered for thrombolysis is computed tomography (CT) scanning. It is quick, universally available, virtually all - even very elderly and quite frail patients can be scanned without delay and it is highly costeffective. $^{10}$ More advanced imaging methods, such as magnetic resonance imaging (MRI) scanning and perfusion or angiography studies, may give much greater information. The Cochrane systematic review did not find any evidence that the extra time, effort and potential risk of these investigations materially altered the response to $\mathrm{rt}-\mathrm{PA} .{ }^{4}$ In summary, there is no evidence yet that patients over $\mathbf{8 0}$ are a group particularly likely to be better selected by more advanced imaging than any other age group.

\section{Is there a case for further randomised controlled trials?}

Reviewing the non-randomised studies of thrombolysis in the over-80s, the authors concluded that the imbalances in baseline variables and lack of randomisation meant there was clearly a need for reliable randomised evidence on the balance of risk and benefit. ${ }^{7}$ They concluded: 'For stroke patients aged over 80 , it is safe and reasonable to include such patients in randomised placebo controlled trials.'7 There are two ongoing trials of thrombolysis with tPA versus control which are including patients aged $>80$ years. The International Stroke Trial (www.ist3.com) has no upper age limit and seeks to recruit 3,100 patients with acute ischaemic stroke by mid-201I." To date, the trial has recruited more than 2,000 patients (almost I,000 of whom are aged over 80 ). The trial will therefore provide unique and reliable data on the balance of risk and benefit in this particular age group. IST-3 expects to report its results 
in early 2012. There is an additional Italian trial called TESPI which seeks to recruit 600 patients aged over 80 within three hours of onset of an acute ischaemic stroke, and which has recruited 36 patients at the time of writing (Danilo Toni, personal communication).

\section{Conclusion}

While it may be acceptable to treat the occasional highly selected patient aged over 80 , routine use of thrombolytic therapy in patients over 80 'off label' is to be avoided until more reliable randomised trial evidence is available. Of course, some patients may not wish to be exposed to the risk of treatment and others may have a very clear view that they wish to be treated whatever the risks (and despite the lack of direct evidence). For the remainder, where both the patient and the doctor are uncertain about the value of thrombolytic therapy, recruitment into one of the ongoing trials is ethical and to be encouraged.

\section{REFERENCES}

I Lees KR, Ford GA, Muir KW, et al; the SITS-UK Group Thrombolytic therapy for acute stroke in the United Kingdom: experience from the safe implementation of thrombolysis in stroke (SITS) register. QJM 2008; 101:863-9. doi:10.1093/qjmed/ henI02

2 Choi $\mathrm{H}$. Thrombolysis in acute ischaemic stroke: example of a health divide? BMJ 2010; 340:c45. doi:10.1136/bmj.c45

3 Perls T. Health and disease in people over 85. BMJ 2009; 339:b47I 5. doi:10.1I36/bmj.b47I5

4 Wardlaw JM, Murray V, Berge E et al. Thrombolysis for acute ischaemic stroke. Cochrane Database Syst Rev 2009; 4:CD0002I3.

5 Sanossian N, Ovbiagele B. Prevention and management of stroke in very elderly patients. Lancet Neurol 2009;8: 103 I-4I.doi:I0.10161 SI474-4422(09)70259-5

6 Hacke W, Donnan G, Fieschi C et al. Association of outcome with early stroke treatment: pooled analysis of ATLANTIS, ECASS, and NINDS rt-PA stroke trials. Lancet 2004; 363:768-74. doi:I0.1016/ SOI40-6736(04)I5692-4

7 Engelter ST, Bonati LH, Lyrer PA. Intravenous thrombolysis in stroke patients of $\geq 80$ versus $<80$ years of age - a systematic review across cohort studies. Age Ageing 2006; 35:572-80. doi: I0.1093/ageing/afl I04

8 Foerch C, Sitzer M, Steinmetz $\mathrm{H}$ et al; Arbeitsgruppe Schlaganfall Hessen (ASH). Future demographic trends decrease the proportion of ischemic stroke patients receiving thrombolytic therapy: a call to set-up therapeutic studies in the very old. Stroke 2009; 40: | 900-2. doi:I0.II6I/STROKEAHA.108.53106 I

9 Reed SD, Cramer SC, Blough DK et al. Treatment with tissue plasminogen activator and inpatient mortality rates for patients with ischemic stroke treated in community hospitals. Stroke 200I; 32: 1832-40.

10 Wardlaw JM, Keir SL, Seymour J et al. What is the best imaging strategy for acute stroke? Health Technol Assess 200I; 8:I-I80.

II Sandercock P, Lindley R, Wardlaw J et al; the IST. The third international stroke trial (IST-3) of thrombolysis for acute ischaemic stroke. Trials 2008; 9:37.

GA Ford

A quarter of all admitted stroke patients in developed countries are aged over 80 years. This proportion will increase over the next 20 years because of three factors: changes in population demographics, improved prevention of stroke in middle-aged individuals and improved survival of patients after first-ever stroke. Stroke in this age group is associated with a high mortality and substantial disability, with only one fifth having a reasonable or good outcome even with the benefits of stroke unit care.' Hence providing treatments that improve the likelihood of a good outcome in this age group is a high priority in the provision of stroke care.

Because of arbitrary age limits in place in the European Stroke Thrombolysis trials (European Cooperation Acute Stroke Studies, ECASS, I-III) only 42 patients over 80 years were included in the randomised controlled trials of recombinant tissue plasminogen activator (rt-PA). Hence some argue that this age group should not be treated until more randomised data are available from further trials. The introduction of an upper age limit in the European licence by the regulatory authority the

European Medicines Agency (EMEA) has also supported overly rigid application of this age limit in current stroke practice. An upper age limit is not in place for any other licensed drug therapy.

Such a rigid view fails to recognise that age is a continuum and that patients over 80 years old are a heterogeneous group comprising, at one end fit, active, healthy individuals who are biologically similar to many patients under 80 years of age and at the other end a group of frail, disabled individuals with multiple comorbidities. The very elderly are more heterogeneous than younger people in respect to their health status, comorbidities, existing drug treatments and views on treatment.

Studies of the effect of ageing on response to drug therapy across a range of therapy areas provide two broad conclusions. Beneficial therapeutic effects of drug treatments may be greater or lesser than those seen in younger patients but are never absent. The risk of serious adverse drug effects usually increases incrementally with age. The introduction of new drug 
therapies is often associated with suggestions that the elderly may not benefit from treatment; suggestions which are not subsequently confirmed. When blood pressure (BP) lowering therapy was first introduced, the prevailing opinion was that BP lowering in those aged over 65 years was hazardous. ${ }^{2}$ Even after subsequent trials in the elderly demonstrated benefit, a meta-analysis in the 1990s of BP lowering in the very elderly suggested that although stroke incidence was decreased by the same relative risk ratio as in younger patients, mortality might be increased. ${ }^{3}$ No clear biological mechanism was proposed for this but a similar non-significant trend to increased mortality was seen in the HYpertension in the Very Elderly Trial (HYVET) pilot study. ${ }^{4}$ However, the main HYVET study examining the effect of BP lowering in over 80 year olds was stopped early because of a significant reduction in overall mortality. ${ }^{5}$

The treatment effect of intravenous thrombolysis with rt-PA for acute ischaemic stroke administered within three hours of symptom onset is large, with a 14\% increase in the likelihood of a good outcome, even taking into account the $2-3 \%$ of patients who experience symptomatic deterioration due to intracerebral haemorrhage. ${ }^{6}$ Thus to deny this treatment to all over 80 year olds, the risk of symptomatic haemorrhage leading to death or disability would have to increase from $2-3 \%$ to at least $10 \%$, or the efficacy in terms of clinical response would have to decrease to less than $20 \%$ of that seen in the trials.

The robustness of the effect of thrombolysis in the $0-3$ hour window has been demonstrated through two other studies. First, the demonstration of efficacy in the 3-4.5 hour window in ECASS III, ${ }^{7}$ and second, the confirmation, in the phase IV observational study Safe Implementation of Thrombolysis in Stroke (SITS), of the same clinical outcomes and symptomatic haemorrhage rate as seen in the randomised controlled trials in a large cohort of patients in European centres treated with intravenous thrombolysis. ${ }^{8}$ The SITS study also demonstrated that the risk of symptomatic haemorrhage as defined by a type II parenchymal haemorrhage with death or neurological deterioration of four or more point increase in the National Institutes of Health Stroke Scale (NIHSS) was $2 \%$.

Many centres have treated patients over 80 years of age who otherwise meet the licensing criteria and have reported outcomes and symptomatic haemorrhage rates. Comparison of outcomes between older and younger patients does not provide data on the effectiveness of treatment. Older patients have worse outcomes in the trials of stroke unit vs usual medical care, but the benefits of stroke unit care were similar in all age groups. A systematic review of six studies comparing outcomes in 477 patients over 80 years receiving thrombolysis with outcomes in the same centres from patients $<80$ years found that the very elderly on average had more severe strokes.' As in studies of patients not treated with thrombolysis, the outcomes with respect to mortality and good outcome were less good in the very elderly, although a very good outcome was reported in $26 \%$ of over 80 year olds. The reported symptomatic intracerebral haemorrhage rate was not significantly increased ( $5.7 \%$ vs $5.2 \%$ ).

In the SITS database approximately $10 \%$ of patients are $>80$ years, with a slightly higher figure for UK SITS centres (16\%). A preliminary review of outcomes in those over 80 years shows a mortality rate and outcome similar to that reported in the published meta-analysis. The symptomatic haemorrhage rate of $2 \%$ was not significantly greater than the $1.5 \%$ seen in $<80$ year olds. ${ }^{10}$

These data indicate that patients over 80 years meeting other criteria for treatment with thrombolysis within three hours of symptom onset have a similar risk of symptomatic haemorrhage as younger patients. Although age is one of the risk factors for symptomatic intracerebral haemorrhage, stroke severity and blood pressure being the other main predictors, there is probably no age threshold at which the benefits of tissue plasminogen activator (tPA) are lost by an excessive bleeding risk in patients who otherwise meet the licence criteria. Age is one among many factors that need to be considered by the stroke specialist in decision-making about thrombolysis in individual patients. In a previously well, independent individual aged over 80 years who has a stroke of moderate severity with normal BP, the benefits of treatment considerably outweigh the potential risk of symptomatic haemorrhage.

Further randomised controlled trials will provide data on the risks and benefits of thrombolysis in patients treated in later time windows and in patients where clinicians are uncertain of the risks and benefits up to 4.5 hours. Other studies, such as the Desmoteplase in Acute Ischemic Stroke Trial, will determine whether advanced imaging can select patients who are more likely to benefit from thrombolysis in later time windows. However, given that age is only one factor influencing the risk and benefit of thrombolysis, it is not logical or appropriate to design and conduct acute stroke trials only recruiting the over-80-year-old population. Future stroke trials should not include upper age limits in their inclusion criteria.

Non-randomised data offer an underutilised opportunity to determine the safety of thrombolysis in different patient groups when randomised controlled trial data are absent. One example is the recent demonstration that the combination of aspirin and clopidogrel at presentation is associated with an increased risk of symptomatic intracerebral haemorrhage following intravenous thrombolysis." Case control studies from well-defined 
cohorts such as the Virtual International Stroke Trials Archive also offer the opportunity to determine the effectiveness of thrombolysis in different patient groups.

The stroke physician at the side of the patient's trolley in the emergency room would benefit from the availability of bedside decision-making tools that define the likely benefits and risks in individual patients. However, for

\section{REFERENCES}

I Kammersgaard LP, Jørgensen HS, Reith J et al. Copenhagen Stroke Study. Short and long term prognosis for very old stroke patients. The Copenhagen Stroke Study. Age Ageing 2004; 33:149-54. doi:10.1093/ageing/afh052

2 Jackson G, Pierscianowski TA, Mahon W et al. Inappropriate antihypertensive therapy in the elderly. Lancet 1976; $2: 1317-8$ doi:I0.1016/S0I40-6736(76)91970-X

3 Gueyffier F, Bulpitt C, Boissel JP et al. Antihypertensive drugs in very old people: a subgroup meta-analysis of randomised controlled trials. Lancet 1999; 353:793-6. doi:I0.10I6/SOI40. 6736(98)08127-6

4 Bulpitt CJ, Beckett NS, Cooke J et al. Results of the pilot study for the Hypertension in the Very Elderly Trial. J Hypertens 2003; 21:2409-17. doi:I0.1097/00004872-2003 I2000-00030

5 Beckett NS, Peters R, Fletcher AE et al.Treatment of hypertension in patients 80 years of age or older. N Engl J Med 2008; 358: I88798. doi:I0.1056/NEJMoa080I369

6 Wardlaw JM, Murray V, Berge E et al. Thrombolysis for acute ischaemic stroke. Cochrane Database Syst Rev 2009; 4:CD0002 I 3

7 Hacke W, Kaste M, Bluhmki E et al. Thrombolysis with alteplase 3 to 4.5 hours after acute ischemic stroke. N Engl J Med 2008; 359:I3।7-29. doi:I0.1056/NEJMoa0804656 most patients including the very old with a potentially disabling stroke deficit without contraindications to thrombolysis, clinicians can be reassured that the riskbenefit ratio is highly favourable.

(This article is based on a talk given in a debate at the UK Stroke Forum in Glasgow, December 2009.)

8 Wahlgren $\mathrm{N}$, Ahmed $\mathrm{N}$, Eriksson $\mathrm{N}$ et al. Multivariable analysis of outcome predictors and adjustment of main outcome results to baseline data profile in randomized controlled trials: Safe Implementation of Thrombolysis in Stroke-MOnitoring STudy (SITS-MOST). Stroke 2008; 39:3316-22. doi:I0.1I6I/ STROKEAHA. 107.510768

9 Engelter ST, Bonati LH, Lyrer PA. Intravenous thrombolysis in stroke patients of $\geq 80$ versus $<80$ years of age - a systematic review across cohort studies. Age Ageing 2006; 35:572-80. doi: I0.1093/ageing/afll 04

10 Ford GA, Larrue V, Ahmed $\mathrm{N}$ et al. Thrombolysis for acute ischemic stroke in patients older than 80 years: results from Sits (Safe Implementation of Thrombolysis in Stroke) Register. In: I5th European Stroke Conference. Cerebrovasc Dis 2006; 2 I (Suppl.4): II62. p. 36. doi: I0.1 I59/000093822

II Diedler J, Ahmed N, Sykora M et al. Safety of intravenous thrombolysis for acute ischemic stroke in patients receiving antiplatelet therapy at stroke onset. Stroke 2010; 41:288-94. doi:I0.I I6I/STROKEAHA.109.559724

\section{EXAM PREPARATION COURSES: Please advise your trainee colleagues}

\section{MRCP PART 2 \\ REVISION COURSE}

\section{9-1I June 2010}

- Three-day exam-orientated course covering many aspects of clinical medicine relevant to the Membership Exam

- Fee: $£ 450$

Tel: 0131 2473649

Fax: 0131 2204393

E-mail: a.fairbairn@rcpe.ac.uk

\section{MRCPCH REVISION COURSES}

VIDEO COURSE

7 June 2010

65 high-quality videos

- Fee: $£ 200$ per course

Tel: 0I3I 2473607

Fax: 013। 2204393

Email: c.gray@rcpe.ac.uk
ETHICS AND COMMUNICATION SKILLS COURSE

9-10 June 2010

- Small-group communication scenarios (including phone conversations)

- Fee: $£ 300$ per course

Tel: 0I3। 2473607

Fax: 0131 2204393

Email: c.gray@rcpe.ac.uk 